\title{
FACTORES ASOCIADOS AL INICIO DE LA LACTANCIA MATERNA PRECOZ
}

\author{
Mirian Solís Rojas ${ }^{1, a}$, Emma Salazar Salvatierra ${ }^{1, b}$, Carmen Del Rocío Huamán Lahura1,c
}

\begin{abstract}
RESUMEN
Objetivo. Analizar los factores asociados al inicio de la lactancia materna precoz del Hospital Nacional Docente Madre Niño "San Bartolomé", Setiembre a Octubre 2017. Materiales y métodos. Estudio observacional, analítico, retrospectivo y transversal. Se encuestó a 191 puérperas que iniciaron precozmente la lactancia materna y 191 controles. El análisis para las variables cualitativas se estimaron frecuencias absolutas y relativas; para las variables cuantitativas se estimaron medias y desviación estándar; para hallar la asociación se usó la prueba Chi-cuadrado y Odds Ratio. Resultados. Factor sociocultural se encontró asociación significativa entre la ocupación estudiante, conocer que no se deforman las mamas y que los bebés no necesitan beber infusiones; y lactancia materna precoz. Factor Institucional se encontró asociación significativa entre el recibir información sobre la lactancia materna en la atención prenatal, la demora en traer al bebé entre 10 a 25 minutos y contar con un ambiente favorable para lactancia materna; y el inicio de la lactancia materna precoz. No se encontró asociación entre el factor obstétrico y neonatal con el inicio de la lactancia materna precoz. Conclusiones. Los factores asociados significativamente al inicio de la lactancia materna precoz fueron: ocupación estudiante, conocer que no se deforman las mamas, conocer que los bebés no necesitan beber infusiones, el tiempo que transcurrió para le entrega del bebé a la madre fue de 10 a 25 minutos y contar con un ambiente favorable para la lactancia materna.
\end{abstract}

Palabras claves: Lactancia Materna; Lactancia materna precoz; Amamantamiento; Factores Lactancia Materna (Fuente: DeCS BIREME).

\section{FACTORS ASSOCIATED WITH THE BEGINNING OF PREMATURE BREASTFEEDING}

\begin{abstract}
Objective. To analyse the factors associated with the initiation of early breastfeeding of the National Mother Teaching Hospital "San Bartolomé", September to October 2017. Material and methods. Observational, analytical, retrospective and crosssectional study. We surveyed 191 women cases who started early and 191 controls. Regarding the analysis of the data, absolute and relative frequencies were estimated for the qualitative variables, and in the case of the quantitative variables, means and standard desviation were estimated; to find the association, the Chi-square test was used and Odds Ratio test. Results. Sociocultural factors, an association was found for student occupation, non-deformity of the breasts due to breastfeeding, the use of infusions to strengthen the stomach) with the on set of breastfeeding. Institutional results, an association was found between receiving information about breastfeeding in prenatal care, the delay in bringing the baby between 10 and 25 minutes and have a favorable environment for breastfeeding with the beginning of breastfeeding. No association was found between the obstetric factor, the neonatal factor and the beginning of early breastfeeding. Conclusion. The factors significantly associated with the start of early breastfeeding were: student occupation, .Know that non-deformity of the breasts, know that babies do not need to drink fusions, the time it takes to bring the baby from 10 to 25 minutes and have a favorable environment for breastfeeding.
\end{abstract}

Keywords: Breastfeeding; Early breastfeeding; Factors breastfeeding(Source: MeSH NLM).

\section{INTRODUCCIÓN}

Las tasas de inicio precoz de la lactancia materna, oscila entre el $17 \%$ en Europa Oriental y el $50 \%$ en los países de América Latina ${ }^{1,2}$.

En el Perú según ENDES 2016, el 50,5\% de niñas y niños empezó a lactar dentro de la primera hora de nacido, esta cifra ascendió a 93,9\% dentro del primer día ${ }^{3}$. Getnet M y Col. (2017) en su estudio realizado en Etiopía encontraron que el $42.6 \%$ de las madres tenían atención prenatal y más de la mitad dieron a luz a sus bebés en casa (56.3\%). Los factores de predicción para el inicio precoz de la lactancia materna de fuerte significación fueron la etnia, la propiedad de la casa, número de hijos vivos, atención prenatal y lugar de nacimiento ${ }^{4}$.

\footnotetext{
Departamento de Obstetricia de la Facultad de Medicina de la UNMSM. Lima-Perú.

a Licenciada en Obstetricia. Doctora en Salud Pública. Docente Principal del Departamento Académico de Obstetricia de la Facultad de Medicina de la UNMSM. Docente investigadora Universidad Privada TELESUP.

b Licenciada en Obstetricia. Magister en Educación. Directora del Departamento Académico de Obstetricia de la Facultad de Medicina de la UNMSM. Docente Principal del Departamento Académico de Obstetricia de la Facultad de Medicina de la UNMSM.

Licenciada en Obstetricia. Serumista en el C.A.P. I Ocallí. Amazonas.
}

Citar como: Solís M, Salazar E, Huamán CDR. Factores asociados al inicio de la lactancia materna precoz. Rev Peru Investig Matern Perinat 2018; 7(2):31-6. https://doi.org/10.33421/inmp.2018115

Recibido: 07-12-18 Aprobado: 30-12-18 
Girard L et al. (2016) en su estudio comparativo Francia y Canadá, encontraron que las tasas de iniciación de la lactancia fueron similares en todas las cohortes, ligeramente por encima del $70 \%$, donde la educación superior de la madre, el no fumar y el ser extranjeras se asociaron significativamente con el inicio de la lactancia precoz $^{5}$

Adhikari M y Col. (2014), Nepal, observaron que de un total de 2861 mujeres, el $70.5 \%$ de las madres que dieron a luz en centros de salud iniciaron la lactancia materna dentro de la primera hora después del parto, en comparación con el $64 \%$ de las madres que dieron a luz en el hogar 6 .

Martínez J y Delgado M (2013) realizaron un estudio en Andalucía, encontrando que el $29.34 \%$ de las madres iniciaron de forma precoz la lactancia materna antes de la primera hora de vida y la educación materna se asocia al inicio precoz de la lactancia materna (ORa $=1.78$, IC 95\% $=1.14-2.78)$ y al mantenimiento de esta $(\mathrm{ORa}=1.73, \mathrm{IC}$ $95 \%=1.15-2.61)^{7}$

Gilany E, Sarraf B y Wehady A (2012) realizaron un estudio en Arabia Saudita, en donde encontraron que de 906 neonatos estudiados, el $91.9 \%$ fueron amamantados, el $8.1 \%$ nunca fueron amamantados y sólo el $11.4 \%$ recibió amamantamiento a tiempo (dentro de 1 hora después del nacimiento). La lactancia oportuna era más probable en las madres con residencia rural, de segunda y tercera paridad, sin problemas de mama, con bebés de peso promedio, parto vaginal espontáneo, atendidos en hospitales gubernamentales o centros de atención primaria de salud, con bebés no ingresados en la unidad de cuidados neonatales, entregados en instalaciones que practican el alojamiento conjunto y no proporcionan alimentos pre lácteos a los bebés ${ }^{8}$.

Quispecondori J y Rivas R (2012) realizó un estudio en Lima, encontrando que los programas de preparación para el parto, la información previa sobre la lactancia materna, la estadía junto al recién nacido inmediatamente después del parto, el parto con acompañante y la decisión previa al parto de dar lactancia materna exclusiva a su recién nacido se asocian significativamente con el inicio de Lactancia Materna Precoz ${ }^{9}$.

El objetivo de la presente investigación fue analizar los factores asociados al inicio de la lactancia materna precoz del Hospital Nacional Docente Madre Niño "San Bartolomé", Setiembre a Octubre del 2017.

\section{MATERIALES Y MÉTODOS}

Investigación observacional, analítica, retrospectiva y transversal. El diseño fue de casos y controles. Población:
641 puérperas inmediatas de parto vaginal atendidas en el Hospital Nacional Docente Madre Niño "San Bartolomé" durante los meses de setiembre a octubre del 2017. Muestra: 382 puérperas inmediatas de parto vaginal, se estimó según la fórmula de comparación de proporciones, se conformaron dos grupos de estudio: caso (puérperas inmediatas que iniciaron la lactancia durante la primera hora de vida) y control (puérperas inmediatas que iniciaron la lactancia después de la primera hora de vida). El muestreo fue no probabilístico consecutivo siempre y cuando se cumplieran con los criterios de selección. Instrumento de recolección de datos: Se elaboró una encuesta conformada por 27 preguntas, la cual fue validada por juicio de siete (07) expertos y una prueba piloto a 20 puérperas para estimar la confiabilidad del instrumento. Técnicas para el procesamiento de los datos: En cuanto al análisis de los datos, para las variables cualitativas se estimaron frecuencias absolutas y relativas (porcentajes), y en el caso de las variables cuantitativas se estimaron medias y desviación estándar; para hallar la asociación se usó la prueba Chi-cuadrado, además de la prueba de Odds Ratio. Aspectos éticos: El proyecto fue sometido ante el Comité de Ética del Hospital Nacional Docente Madre Niño "San Bartolomé". Se aplicó consentimiento informado y siguiendo el principio de confidencialidad no se divulgaron los nombres de las personas que aceptaron ser parte del estudio.

\section{RESULTADOS}

De las características del inicio de la lactancia materna precoz se estudió: la intención de dar de lactar desde antes del parto, la decisión de dar lactancia materna dentro de la primera hora de nacido y el tiempo que dio de lactar durante la primera hora postparto. Obteniendo que, el $56 \%$ tiene la intención de iniciar la lactancia antes del parto; el $97.6 \%$ tiene la decisión de dar de lactar dentro de la primera hora de nacido el bebé; el $26.4 \%$ dio de lactar dentro de los 15 a 30 minutos postparto y el $22.3 \%$ antes de los 15 minutos.

Los factores socioculturales significativos para el inicio de la lactancia materna precoz fueron: la ocupación "estudiante", el pensar que la lactancia materna no deforma las mamas y el pensar que los bebés no necesitan infusiones para fortalecer el estómago; mientras que los que fueron significativos para el no inicio de lactancia materna precoz fueron: la ocupación "trabajo dependiente", el pensar que la lactancia materna produce dolor, el pensar que se debe esperar hasta que salga la leche de color blanco y el consumo de tabaco. (ver tabla 1).

Respecto a los factores institucionales, se observó asociación significativa para el inicio de la lactancia materna precoz con recibir información sobre lactancia 
Tabla 1. Factores socioculturales asociados al inicio de la lactancia materna precoz del Hospital Nacional Docente Madre Niño "San Bartolomé", setiembre a octubre del 2017.

\begin{tabular}{|c|c|c|c|c|c|c|c|}
\hline & & \multicolumn{4}{|c|}{ Inicia lactancia materna precoz } & \multirow{3}{*}{$\mathbf{p}$} & \multirow{3}{*}{ OR } \\
\hline \multirow{2}{*}{\multicolumn{2}{|c|}{ Factores socioculturales }} & \multicolumn{2}{|c|}{$\begin{array}{c}\text { Grupo Casos } \\
\text { (Si) }\end{array}$} & \multicolumn{2}{|c|}{$\begin{array}{l}\text { Grupo Control } \\
\text { (No) }\end{array}$} & & \\
\hline & & $\mathbf{N}$ & $\%$ & $\mathbf{N}$ & $\%$ & & \\
\hline \multirow{3}{*}{ Edad } & 18-20 años & 27 & $14.1 \%$ & 19 & $9.9 \%$ & \multirow{3}{*}{$0.394+$} & \multirow{3}{*}{ - } \\
\hline & 21-34 años & 138 & $72.3 \%$ & 141 & $73.8 \%$ & & \\
\hline & Más de 34 años & 26 & $13.6 \%$ & 31 & $16.2 \%$ & & \\
\hline \multirow{3}{*}{ Procedencia } & Costa & 121 & $63.4 \%$ & 129 & $67.5 \%$ & \multirow{3}{*}{$0.161^{*}$} & - \\
\hline & Sierra & 36 & $18.8 \%$ & 41 & $21.5 \%$ & & - \\
\hline & Selva & 34 & $17.8 \%$ & 21 & $11.0 \%$ & & - \\
\hline \multirow{4}{*}{ Ocupación } & Ama de casa & 138 & $72.3 \%$ & 143 & $74.9 \%$ & $0.562^{*}$ & - \\
\hline & Estudiante & 20 & $10.5 \%$ & 6 & $3.1 \%$ & $0.003^{*}$ & $3,61(1.41-9.19)$ \\
\hline & Trabajo dependiente & 7 & $3.7 \%$ & 25 & $13.1 \%$ & $0.000^{*}$ & \multirow[t]{2}{*}{$0.25(0.11-0,60)$} \\
\hline & Trabajo independiente & 26 & $13.6 \%$ & 17 & $8.9 \%$ & $0.116^{*}$ & \\
\hline \multirow{4}{*}{ Grado de instrucción } & Primaria & 6 & $3.1 \%$ & 8 & $4.2 \%$ & \multirow{4}{*}{$0.959^{*}$} & - \\
\hline & Secundaria & 129 & $67.5 \%$ & 128 & $67.0 \%$ & & \\
\hline & Superior Técnico & 30 & $15.7 \%$ & 29 & $15.2 \%$ & & \\
\hline & Superior universitario & 26 & $13.6 \%$ & 26 & $13.6 \%$ & & \\
\hline \multirow{4}{*}{ Tipo de familia cuando era una niña } & Mamá, papa e hijos & 110 & $57.6 \%$ & 104 & $54.5 \%$ & \multirow[t]{4}{*}{$0.146^{*}$} & \multirow[t]{4}{*}{-} \\
\hline & $\begin{array}{l}\text { Matrimonio e hijo de } \\
\text { uno de los cónyuges }\end{array}$ & 3 & $1.6 \%$ & 0 & $0.0 \%$ & & \\
\hline & Mamá o papá con hijos & 50 & $26.2 \%$ & 47 & $24.6 \%$ & & \\
\hline & $\begin{array}{l}\text { Abuelos, padres, tíos } \\
\text { e hijos }\end{array}$ & 28 & $14.7 \%$ & 40 & $20.9 \%$ & & \\
\hline \multirow{2}{*}{$\begin{array}{l}\text { La lactancia materna no deforma las } \\
\text { mamas. }\end{array}$} & Si & 128 & $67.0 \%$ & 109 & $57.1 \%$ & \multirow{2}{*}{$0.045^{*}$} & \multirow[t]{2}{*}{$1,53(1,01-2,32)$} \\
\hline & No & 63 & $33.0 \%$ & 82 & $42.9 \%$ & & \\
\hline \multirow{2}{*}{ El dar lactancia materna produce dolor. } & Si & 34 & $17.8 \%$ & 58 & $30.4 \%$ & \multirow{2}{*}{$0.004^{*}$} & \multirow[t]{2}{*}{$0,50(0,31-0,80)$} \\
\hline & No & 157 & $82.2 \%$ & 133 & $69.6 \%$ & & \\
\hline \multirow{2}{*}{$\begin{array}{l}\text { La lactancia materna debe esperar hasta } \\
\text { que salga la leche de color blanco }\end{array}$} & $\mathrm{Si}$ & 6 & $3.1 \%$ & 15 & $7.9 \%$ & \multirow{2}{*}{$0.043^{*}$} & \multirow[t]{2}{*}{$0,38(0,14-1,00)$} \\
\hline & No & 185 & $96.9 \%$ & 176 & $92.1 \%$ & & \\
\hline \multirow{2}{*}{$\begin{array}{l}\text { Los bebes no necesitan infusiones para } \\
\text { fortalecer el estómago. }\end{array}$} & Si & 42 & $22.0 \%$ & 17 & $8.9 \%$ & \multirow{2}{*}{$0.000^{*}$} & $2,89(0,58-5,28)$ \\
\hline & No & 149 & $78.0 \%$ & 174 & $91.1 \%$ & & \\
\hline & $\mathrm{Si}$ & 2 & $1.0 \%$ & 9 & $4.7 \%$ & & \\
\hline comsurio de lavaco & No & 189 & $99.0 \%$ & 182 & $95.3 \%$ & 0.032 & $0,21(0,00-1,00)$ \\
\hline Total & & 191 & $100.0 \%$ & 191 & $100.0 \%$ & & \\
\hline
\end{tabular}

U de Mann-Whitney (+)

Chi cuadrado $\left(^{*}\right)$

materna durante la atención prenatal, el tiempo que demoraron en llevar al bebé con su madre y si tienen opinión favorable en cuanto al ambiente donde se da de lactar; en cambio, hubo asociación significativa para el no inicio de la lactancia materna precoz con el recibir información sobre lactancia materna en Psicoprofilaxis
Obstétrica y la administración de analgesia de parto. (ver tabla 2).

Respecto a los factores obstétricos, se estudiaron: la posición del parto, episiotomía y desgarro perineal, sin embargo se identificó asociación significativa entre la 
Tabla 2. Factores institucionales asociados al inicio de la lactancia materna precoz del Hospital Nacional Docente Madre Niño "San Bartolomé", setiembre a octubre del 2017.

\begin{tabular}{|c|c|c|c|c|c|c|c|}
\hline \multirow{3}{*}{ Factores Institucionales } & & \multicolumn{4}{|c|}{ Inicia lactancia materna precoz } & \multirow{3}{*}{$\mathbf{p}$} & \multirow{3}{*}{ OR } \\
\hline & & \multicolumn{2}{|c|}{ Grupo casos } & \multicolumn{2}{|c|}{$\begin{array}{l}\text { Grupo Control } \\
\text { (No) }\end{array}$} & & \\
\hline & & $(\mathrm{Si})$ & $\begin{array}{l}\text { Grupo } \\
\text { control }\end{array}$ & $\mathbf{N}$ & $\%$ & & \\
\hline \multirow{2}{*}{$\begin{array}{l}\text { Reciben información sobre lactancia materna } \\
\text { durante la atención prenatal (APN) }\end{array}$} & $\mathrm{Si}$ & 110 & $57.6 \%$ & 88 & $46.1 \%$ & \multirow{2}{*}{$0.024^{*}$} & \multirow{2}{*}{$1.59(1.06-2.38)$} \\
\hline & No & 81 & $42.4 \%$ & 103 & $53.9 \%$ & & \\
\hline \multirow{2}{*}{$\begin{array}{l}\text { Reciben información sobre lactancia materna en las } \\
\text { sesiones de Psicoprofilaxis Obstétrica }\end{array}$} & $\mathrm{Si}$ & 50 & $26.2 \%$ & 85 & $44.5 \%$ & \multirow{2}{*}{$0.002^{*}$} & \multirow{2}{*}{$0.44(0.29-0.68)$} \\
\hline & No & 141 & $73.8 \%$ & 106 & $55.5 \%$ & & \\
\hline \multirow{2}{*}{ Se les administra analgesia de parto. } & $\mathrm{Si}$ & 13 & $6.8 \%$ & 34 & $17.8 \%$ & \multirow{2}{*}{$0.001^{*}$} & \multirow{2}{*}{$0.34(0.17-0.66)$} \\
\hline & No & 178 & $93.2 \%$ & 157 & $82.2 \%$ & & \\
\hline \multirow{2}{*}{ Se realiza contacto piel a piel. } & $\mathrm{Si}$ & 175 & $91.6 \%$ & 165 & $86.4 \%$ & \multirow{2}{*}{$0.102^{*}$} & \multirow{2}{*}{ - } \\
\hline & No & 16 & $8.4 \%$ & 26 & $13.6 \%$ & & \\
\hline \multirow{4}{*}{$\begin{array}{l}\text { Tiempo que demoraron en llevar al bebé con su } \\
\text { madre. }\end{array}$} & $10-25 \mathrm{~min}$. & 97 & $50.8 \%$ & 15 & $7.9 \%$ & $0.000+$ & $12.11(6.65-22.03)$ \\
\hline & $26-45 \mathrm{~min}$ & 76 & $39.8 \%$ & 14 & $7.3 \%$ & $0.000+$ & $8.36(4.51-15.48)$ \\
\hline & $46-60 \min$ & 14 & $7.3 \%$ & 37 & $19.4 \%$ & $0.001+$ & $0.33(0.17-0.63)$ \\
\hline & Más de $1 \mathrm{hr}$. & 4 & $2.1 \%$ & 125 & $65.4 \%$ & $0.000+$ & $0.01(0.00-0.03)$ \\
\hline \multirow{2}{*}{$\begin{array}{l}\text { Tienen opinión favorable en cuanto al ambiente } \\
\text { donde se da de lactar. }\end{array}$} & $\mathrm{Si}$ & 162 & $84.8 \%$ & 144 & $75.4 \%$ & \multirow{2}{*}{$0.021^{*}$} & \multirow{2}{*}{$1.82(1.09-3.05)$} \\
\hline & No & 29 & $15.2 \%$ & 47 & $24.6 \%$ & & \\
\hline \multirow{2}{*}{ Horario del parto } & G. diurna & 106 & $55.5 \%$ & 91 & $47.6 \%$ & \multirow{2}{*}{$0.125^{\star}$} & \\
\hline & G. Nocturna & 85 & $44.5 \%$ & 100 & $52.4 \%$ & & \\
\hline Total & & 191 & $100.0 \%$ & 191 & $100.0 \%$ & & \\
\hline
\end{tabular}

U de Mann-Whitney (+)

Chi cuadrado $\left({ }^{*}\right)$

posición acostada del parto y el no inicio de la lactancia materna precoz. Existe $39 \%$ de probabilidad para el no inicio de lactancia materna precoz en pacientes cuya posición del parto es acostada.

No se encontró asociación entre la episiotomía, el desgarro perineal y el inicio de la lactancia materna precoz.

En cuanto a los factores neonatales, se estudiaron: Apgar al minuto, cinco minutos, el sexo y el peso del bebé, sin embargo se identificó asociación significativa entre el inicio de la lactancia materna precoz y el peso del bebé mayor o igual a 4000 gramos.

No se evidenció asociación entre Apgar al minuto, a los cinco minutos, el sexo del bebé y el inicio de la lactancia materna precoz.

\section{DISCUSIÓN}

La lactancia materna es el acto de alimentar a un niño (a) con los nutrientes óptimos para su desarrollo y crecimiento.
Asimismo, es una actividad que se promueve en distintas instituciones de salud por los múltiples beneficios tanto para la madre como para los niños.

El ser estudiante tiene 3,61 veces más probabilidad de tener un inicio precoz en la lactancia materna, tal vez porque la mayoría de puérperas estudiantes tienen su primer embarazo y recién experimentan un vínculo con su bebé, teniendo en cuenta lo recomendado en la institución.

Dentro de las creencias, el pensar que los bebés no necesitan beber infusiones (manzanilla, hinojo, anís, etc.), tuvo dos veces mayor probabilidad para el inicio de la lactancia materna de manera temprana. Similar al estudio de Gilany E y col, donde se encontró una mayor probabilidad (1,7 veces) de lactancia materna en las mujeres que manifestaron no proporcionar alimentos pre lácteos $u$ otras bebidas ${ }^{8}$. Asimismo, en el estudio de Khanal $V$ et al., se halló que el hecho de pensar no suministrarles alimentos prelactales a los bebés tuvo una mayor probabilidad de iniciar el amamantamiento dentro de la primera hora después del nacimiento ${ }^{10}$. 
Con respecto a los factores institucionales, recibir información sobre la lactancia materna en la atención prenatal se asoció de manera significativa con el inicio de la lactancia materna precoz $(p=0,024)$, existiendo 1.59 veces mayor probabilidad de inicio de lactancia materna temprana en las puérperas que reciben información, similar a la investigación de Vieira et Al. , quiénes encontraron que la orientación prenatal sobre las ventajas de la lactancia materna se asoció con el inicio de la lactancia materna en la primera hora después del nacimiento $(p=0,000)^{11}$.

Asimismo, se tuvo que el tiempo que demoran en traerle a su bebé después del parto, tuvo una asociación significativa con el inicio de la lactancia materna precoz, encontrándose una mayor asociación entre el inicio precoz de la lactancia y la demora de llevar al bebé menor a 45 minutos $(p=0.000)$. De manera similar se tuvo en el estudio de Quispecondori J y col., quiénes hallaron que la estadía en Centro Obstétrico de las madres junto a sus recién nacidos, está asociado significativamente con el inicio de la lactancia materna precoz $(p=0,007)^{9}$. Esto demuestra que mientras más temprana es la entrega del recién nacido a su madre, se puede llevar a cabo la lactancia materna precoz, además de los beneficios que aporta.

En cuanto a los factores obstétricos asociados para el inicio de la lactancia materna precoz se observó un menor porcentaje del grupo casos (20.9\%) que adoptó la posición acostada al momento del parto, comparado con el grupo control (30.4\%), observándose una asociación significativa $(p=0,035)$ es decir, existe $39 \%$ mayor probabilidad para el no inicio de lactancia materna precoz en las pacientes que adoptan la posición acostada al momento del parto. Esto devela que posiblemente al adoptar la posición acostada, dicha acción la conduzca a que adquiera mayor nivel de dolor y por consiguiente menor predisposición a desear tener una lactancia materna precoz. Asimismo, el hecho de adoptar una posición libre y cómoda para el parto le da más probabilidades a la madre de iniciar la lactancia materna precoz.

Acerca de los factores neonatales asociados para el inicio de la lactancia materna precoz se encontró que las frecuencias de recién nacidos de sexo masculino en el grupo casos $(48.2 \%)$ y control $(46.6 \%)$ eran similares; por tanto, el sexo del neonato no se asoció al inicio de la lactancia materna precoz, lo cual no concuerda con el trabajo de Patel A, et al., quienes encontraron que el sexo masculino tenía significativamente menos probabilidad de iniciar la lactancia materna ${ }^{12}$

Conclusiones: Los factores asociados significativamente al inicio de la lactancia materna precoz en puérpera inmediatas del Hospital Nacional Docente Madre Niño "San Bartolomé" fueron: el pensar que los bebés no necesitan beber infusiones $(p=0.000)$, el tiempo que demoran en traerle al bebé "de 10 a 25 minutos" $(p=0.000)$, y la ocupación "estudiante" $(p=0.000)$. Los factores socioculturales que se asociaron de manera significativa al inicio de la LMP fueron: la ocupación "estudiante" ( $p=0.000)$, el creer que los bebés no necesitan beber infusiones $(p=0.000)$ y el creer que la lactancia materna no deforma las mamas $(p=0.045)$. Los factores institucionales que se asociaron de manera significativa al inicio de la LMP fueron: el tiempo que demoraron en entregar a su bebé después del parto $(p=0.000)$; el creer que hay un ambiente favorable para la lactancia materna en el hospital $(p=0.021)$ y el recibir información sobre la lactancia materna en la atención prenatal $(p=0.024)$. No se encontró asociación significativa entre los factores obstétricos y el inicio de la lactancia materna precoz. No se encontró asociación significativa entre los factores neonatales y el inicio de la lactancia materna precoz.

\section{Financiamiento: Autofinanciado}

Conflicto de interés: Los autores declaran no tener algún conflicto de intereses.

\section{REFERENCIAS BIBLIOGRÁFICAS}

1. World Health Organization. Infant and young child feeding. Fact sheet N`342. Geneve: World Health Organization; 2016. [Access on June 5, 2016]. Available in: http://www.who.int/ mediacentre/factsheets/fs342/en/.

2. Sallam S, Babrs G, Sadek R, Mostafa A. Knowledge, attitude, and practices regarding early start of breastfeeding among pregnant, lactating women and healthcare workers in ElMinia University Hospital. Breastfeed Med [Online magazine]. 2013 [Access on June 5, 2016]; 8(3):312-6. doi: 10.1089/ bfm.2012.0040.

3. Instituto Nacional de Estadística e Informática. Encuesta Demográfica y de Salud Familiar. Perú: INEI; 2016. [Acceso el 04 de agosto del 2016]. Disponible en: https://www.inei. gob.pe/media/MenuRecursivo/publicaciones_digitales/Est/ Lib1433/index.html.

4. Getnet M, Regassa N, Dejenie R, Mengistu Z. Early initiation of breastfeeding among mothers of children under the age of 24 months in Southern Ethiopia. International Breastfeeding Journal [Online Magazine]. 2017 [Access on March 05, 2017]; 12:1. DOI 10.1186/s13006-016-0096-3.

5. Girard L, Coté S, De Lauzon B, Dubois L, Falissard B, Forhan $A$, et al. Factors Associated with Breastfeeding Initiation: A Comparison between France and French-Speaking Canada. PLoS ONE [Online Magazine]. 2016 [Access on March 10, 2017]; 11(11): e0166946. doi: 10.1371/journal.pone.0166946.

6. Adhikari M, Khanal V, Karkee R, Gavidia T. Factors associated with early initiation of breastfeeding among Nepalese mothers: further analysis of Nepal Demographic and Health Survey, 2011. Int Breastfeed J [Online Magazine]. 2014 [Access on March 29, 2017]; 9(1):21. doi: 10.1186/s13006-014-0021-6.

7. Martínez J, Delgado M. El Inicio Precoz de la Lactancia Materna se ve favorecido por la realización de la Educación Maternal. Rev Assoc Bra. [Revista en Internet]. 2013 [Acceso el 21 de marzo del 2017]; 59(3): 254-257. Disponible en: http://dx.doi.org/10.1016/j.ramb.2012.12.001.

8. Gilany E, Sarraf B, Wehady A. Factors associated with timely initiation of breastfeeding in Al-Hassa province, Saudi Arabia. 
Eastern Mediterranean Health Journal [Online Magazine]. 2012 [Access on March 28, 2017]; 18 (3): 205-4. Available in: https://www.ncbi.nlm.nih.gov/pubmed/22574479.

9. Quispecondori J, Rivas R. Factores que se asocian con el inicio de la lactancia materna precoz en puérperas primíparas en el Instituto Nacional Materno Perinatal: noviembre 2011 enero 2012 [Tesis de licenciatura]. Lima: Universidad Nacional Mayor de San Marcos. Facultad de Medicina Humana; 2012. [Acceso el 21 de marzo del 2017]. Disponible en: http:// cybertesis.unmsm.edu.pe/bitstream/cybertesis/3015/1/ Quispecondori_qj.pdf.

10. Khanal, V., Scott, J., Lee, A., Karkee, R, Binns, C. Factors associated with Early Initiation of Breastfeeding in Western Nepal. Int. J. Environ, Res. Public Health [Online Magazine]. 2015 [Access on March 10, 2017]; 12(8):9562-74. doi: 10.3390/ijerph120809562.
11. Vieira T, Vieira G, Giugliani E, Mendes C, Martins C, Silva L. Determinants breastfeeding initiation within the first hour of life in a Brazilian population: cross-sectional study of. BMC Public Health. 2010; 10:760.

12. Patel A, Bucher S, Pusdekar Y, Esamai F, Krebs N, Goudar S, et al. Rates and determinants of early initiation of breastfeeding and exclusive breast feeding at 42 days postnatal in six low and middle-income countries: A prospective cohort study. Reprod Health [Online Magazine]. 2015 [Access on March 20, 2017]; 12(2): S10. doi: 10.1186/1742-4755-12-S2-S10.

\section{Correspondencia:}

Mirian Solís Rojas.

General Clement 891 Pueblo Libre.

E-mail: misolroj2@gmail.com 